\title{
MOTION OF COMPRESSIBLE LIQUIDS IN PIPES
}

\author{
Hikmet Hassanov \\ Azerbaijan Petroleum Research and Project Institute \\ (AzNSETLI) \\ Aga - Neymatullah str. 39 , Baku 370033 - Azerbaijan
}

\begin{abstract}
The hydrodynamic problem of defining the velocity profile of liquid is resolved, assuming for the liquid density to be compressed in accordance with exponential law along coordinate $z$. The continuity equation for this liquid is concluded. Influence of barometric index $\lambda$ on motion character is estimated. Flowrate decrease and coefficient of hydraulic losses for the compressed liquid are found. Principle of quasi - isothermal approximation is established, and the effect of temperature gradient on final result is qualitatively considered
\end{abstract}

\section{INTRODUCTION}

For solving most of hydrodynamic problems, liquid is taken incompressible as simplifying factor. Mathematically, the last condition is expressed like div $v=0$, which from physical point of view means absence of any sources of potential motion of liquid within volume involved, additional to already existing motion and caused by external pressure drop $\partial \mathrm{P} / \partial \mathrm{z}$. However, such an approach is strongly idealized and is not valid in enormous number of real problems, which are of practical interest, for example, for all the technological operations in drilling, liquid motion in pipe with temperature gradient different from zero etc. Finding the velocities profile in above mentioned cases requires already knowledge of non - zero summand proportional to div v. The resolution of such problems connects with mathematical difficulties and accordingly to current information these questions have been studied for restricted cases, namely, in "Boussinesq approximation "[ 1 ]. As a rule in this approach ones involve density small deviation from certain permanent value only [ 2,3 ]. However, for practice it is natural to consider essential variations of liquids ( or gases ) density by coordinate. In the paper offered we consider motion of liquids, densities of which change along pipe by exponential law available in a lot of practical cases $[4,5,7]$.

\section{CONTINUITY EQUATION}

Typical example of such a liquid is drilling mud in borehole annulus. In [ 4 ] it is stated, drilling mud density with account of cuttings not removed from borehole changes by exponential law. Moreover, taking into consideration barometric index $\lambda$ allows us to develop optimal drilling regime from both technological point of view, and economical that [ 5 ]. Except for all, barometric index different from zero essentially changes hydrodynamic characteristics of moving liquid. It clearly follows from continuity equation. How it is known

$$
\frac{\partial \rho}{\partial t}+\operatorname{div}(\rho v)=0
$$


If $\lambda \neq 0$, then there is aggregation of sludge in well and, hence, $\partial \rho / \partial \mathrm{t}$ is not equal to zero also. Let's take into account

$$
\rho(z)=\rho_{0} e^{\lambda z}
$$

where $\rho_{0}$ is mud density in point $z=0$ (in drilling terms this point is called bottomhole of well ); relationship ( 2 ) is valid for liquid isothermal motion or limited drilling interval, when temperature may be proposed as constant. Because $\rho \neq$ const, equation ( 1 ) should be transformed to next form

$$
\frac{\partial \rho}{\partial t}+\rho \operatorname{div} v+v \operatorname{grad} \rho=0
$$

Use of distribution ( 2) gives us

$$
\frac{\partial \rho}{\partial t}=\rho \lambda w, \quad \operatorname{grad} \rho=-\lambda \rho
$$

hereafter it is taken for simplification of calculations, that penetration rate ( bit mechanical velocity ) $\mathrm{w}=\partial \mathrm{z} / \partial \mathrm{t}$ is permanent and directed against the axis $z$ in cylindrical coordinates system as well as uniform radial distribution of sludge, i.e. $\partial \rho / \partial \mathrm{r}=0$. Ultimately, after non - complex mathematical transformations we yield

$$
\operatorname{div} v=(v-w) \lambda
$$

that proves assumption about unique meaning of parameter $\lambda$ for description of compressible liquid made above. Therefore, the summand characterizing hydrodynamic properties of compressible liquid is found by formula ( 3 ). Now, ones are able to solve equation for such a liquid.

\section{FINDING VELOCITY PROFILE FOR COMPRESSIBLE LIQUID}

Let 's consider the simplest case - laminar flow of compressible liquid along horizontal pipe with permanent pressure drop, that is described by equation

$$
\rho \frac{\partial v}{\partial t}=\eta \Delta_{r} v+\beta \text { graddiv } v+\frac{\Delta P}{L}
$$

where next abbreviations are introduced

$$
\Delta_{r}=\frac{\partial^{2}}{\partial r^{2}}+\frac{1}{r} \frac{\partial}{\partial r}, \quad \beta=\frac{\eta}{3}+\xi
$$

$\xi$-volume viscosity [ 6 ]; more general and complicated variant of problem with non permanent pressure drop $\partial \mathrm{P} / \partial \mathrm{z}$ was resolved by us as well, for interested persons we would like to recommend [ 7 ].Taking into consideration relationship ( 3 ) as well as condition

$w \neq w(r)$, we obtain

$$
\operatorname{grad} \operatorname{div} v=\lambda \frac{\partial v}{\partial r}
$$

or ultimately for equation of liquid motion

$$
\rho \frac{\partial v}{\partial t}=\eta \Delta_{r} v+\beta \lambda \frac{\partial v}{\partial r}+\frac{\Delta P}{L}
$$

that should be integrated under the following time and boundary conditions 


$$
v(r, t=0)=v_{0}, v\left(r=R_{1}, t\right)=0, v\left(r=R_{2}, t\right)=0
$$

$R_{1}$ and $R_{2}$ - radii of internal and external cylinders, respectively (in drilling terms $R_{1}$ external radius of drill string, $R_{2}$ - radius of well ). For solving the equation ( 5 ), let's make substitution of required function

$$
v(r, t)=u(r, t)+\Delta P \cdot \alpha(r) / L \eta
$$

here $\alpha(\mathrm{r})$ - certain correlation function to be defined below. Having found expressions for all the derivatives of $v(\mathrm{r}, \mathrm{t})$ from the last substitution and inserting them into the equation ( 5 ), we get

$$
\rho \frac{\partial u}{\partial t}=\eta\left\{\Delta_{r} \alpha(r)+\frac{\Delta P}{\eta L} \Delta_{r} \alpha(r)\right\}+\beta \lambda \frac{\partial u}{\partial r}+\frac{\beta \lambda \Delta P d \alpha(r)}{\eta L}+\frac{\Delta P}{L}
$$

Demand for correlation function $\alpha(\mathrm{r})$ to be justified the next equation

$$
\frac{\Delta P}{L} \Delta_{r} \alpha(r)+\frac{\beta \lambda}{\eta} \frac{\Delta P}{L} \frac{d \alpha(r)}{d r}+\frac{\Delta P}{L}=0
$$

in this case the following equation should be valid for $u(r, t)$

$$
\rho \frac{\partial u}{\partial t}=\eta \Delta, u+\beta \lambda \frac{\partial u}{\partial r}
$$

Under conditions ( 6 ) the equation ( 8 ) can be solved by variables division method. Representing function $u(r, t)$ as

$$
u(r, t)=u_{i} \cdot u_{r}
$$

we yield two independent equations

$$
\frac{d u_{t}}{d t}=-\frac{\tau}{\rho} d t
$$

and

$$
\eta \Delta_{r} u_{r}+\beta \lambda \frac{d u_{r}}{d r}+\tau u_{r}=0
$$

where $\tau$ is accepted as empirical parameter showing relaxation properties of liquid involved. Solution of ( $9 a$ ), obviously, is the exponentially decreasing function of type

$$
u_{t}=C e^{-\tau t / \rho}
$$

Equation ( $9 \mathrm{~b}$ ) is the confluent hypergeometric equation. For considered case it can be proved, that solution of the last comes to cylindrical functions. Indeed, after appropriate transformations solution of the equation ( $9 \mathrm{~b}$ ) is expressed like [8]

$$
u_{r}=r^{-1 / 2} e^{-\sigma r / 2} y\left(-\frac{\sigma}{2 \theta} ; 0 ; \theta r\right)
$$

where next notes are used

$$
\sigma=\beta \lambda / \eta, \quad \theta=\sqrt{\sigma^{2}-4 \tau / \eta}
$$

$y\left(-\frac{\sigma}{2 \theta} ; 0 ; \theta r\right)$ - confluent hypergeometric function (more detailly this function is considered in [9]). 
Analysis of liquid physical parameters gives that for any really relaxing liquid $\tau$ is sufficiently large magnitude; at least there is approximation $\sigma^{2}<4 \tau / \eta$. That's why, the last solution may be written as

$$
u_{r}=r^{-1 / 2} e^{-\sigma r / 2} y\left(0 ; 0 ; 2 \theta_{1} i r\right) ; \theta_{1}=\sqrt{\tau / \eta}
$$

that due to properties of hypergeometric functions [ 9 ] is substituted for

$$
u_{r}=\left(2 \theta_{1} i\right)^{1 / 2} e^{i \theta_{1} r / 2} e^{-\sigma r / 2} J_{0}\left(\theta_{1} r\right)
$$

Solution $\mathrm{u}_{\mathrm{r}}$ in the form ( 10 ) is complex; calculating its module, we ultimately get

$$
u_{r}=\sqrt{2 \theta_{1}} e^{-\sigma r / 2} J_{0}\left(\theta_{1} r\right)
$$

here $J_{o}\left(\theta_{l} r\right)$ - Bessel function of the first kind. Hence, for non - stationary summand of required velocities profile it can be written down

$$
u(r, t)=C \sqrt{2 \theta_{1}} e^{-\sigma r / 2} J_{0}\left(\theta_{1} r\right) e^{-\tau t / \rho}
$$

\section{FINDING THE CORRELATION FUNCTION}

The solution of ( 11 ) is valid only, if for function $\alpha(r)$ equation ( 7 ) is justified, i.e.

$$
r \alpha^{\prime \prime}+(1+\sigma r) \alpha^{\prime}+r=0
$$

For solving the last, let's take $\mathrm{r} \alpha^{\prime}(\mathrm{r})=\psi(\mathrm{r})$, after that we get the differential equation of the first order relative to $\psi(r)$

$$
\psi^{\prime}(r)+\sigma \psi(r)+r=0
$$

Function $\psi(\mathrm{r})$, obviously, have a form

$$
\psi(r)=e^{-\sigma r}\left\{C_{1}-\int r e^{\sigma r} d r\right\}=C_{1} e^{-\sigma r}-\frac{1+\sigma r}{\sigma^{2}}
$$

Accordingly to this, for function $\alpha(\mathrm{r})$ the next expression appears

$$
\alpha(r)=C_{1}(\ln r-\sigma r)-\frac{\ln r}{\sigma^{2}}-\frac{r}{\sigma}+C_{2}
$$

here $\mathrm{C}_{1}$ and $\mathrm{C}_{2}$ - certain constants, that should be found from boundary conditions ( 6 ). During calculation for function ( 12 ) the approach $\sigma \mathrm{r} \ll 1$ was used, since for involved motion process volume viscosity $\xi$ is sufficiently small magnitude, so parameter $\beta$ is approximately equal to $\eta / 3$; under these conditions coefficient $\sigma$ value is close to that of index $\lambda[4]$. Because of this reason summands proportional to the second and higher degrees of $\sigma$ are neglected. Constants $C_{1}$ and $C_{2}$ defined from conditions the ( 6 ) prove to be

$$
C_{1}=\frac{1}{\sigma^{2}}\left[\frac{\ln \frac{R_{2}}{R_{1}}+\sigma \cdot \Delta R}{\ln \frac{R_{2}}{R_{1}}-\sigma \cdot \Delta R}\right]
$$




$$
C_{2}=C_{1}\left(\sigma R_{1}-\ln R_{1}\right)+\frac{\ln R_{1}}{\sigma^{2}}+\frac{R_{1}}{\sigma} \quad ; \quad \Delta R=R_{2}-R_{1}
$$

By physical matter correlation function gives stationary value of liquid velocity (at $t \rightarrow \infty$ ) as function of $\mathrm{r}$. Analysis of (12) shows, $\mathrm{d} \alpha(\mathrm{r}) / \mathrm{dr}$ undergoes sign change, i.e. in dependence $\alpha(r)$ there is maximum to be defined by the following relationship

$$
r_{e x t}=\frac{\Delta R}{\ln \frac{R_{2}}{R_{1}}+\sigma \cdot \Delta R}
$$

As it follows from relationship the (13) $r_{\text {ext }}$ is not placed inevitably in geometrical middle between radii $R_{1}$ and $R_{2}$. Reason of this is, under compressibility condition of liquid

$(\lambda \neq 0)$ additional velocity gradient connected with $\partial v / \partial r$ arises. This gradient is directed radially and naturally violates the symmetry of velocities profile in full accordance to Segre - effect ( more detailly the effect is described in [ 10,11 ] ). Moreover, it can be shown that for compressible liquid velocities profile will change sharper in dependence of $r$ than for incompressible one. The last circumstance leads ultimately to deterioration of flow hydraulic characteristics. For instance, for stationary flow regime, when liquid velocity $u_{\text {stat }}$ is defined by

$$
u_{\text {stat }}=\frac{\Delta P}{L \eta} \alpha(r)
$$

(since summand $\mathrm{u}(\mathrm{r}, \mathrm{t})$ in this case runs asymptotically to zero ) flowrate between two cylindrical pipes will be equal to

$$
Q=A_{1}\left(R_{2}^{3}-R_{1}^{3}\right)+A_{2}\left(R_{2}^{2}-R_{1}^{2}\right)-A_{3} \ln \frac{R_{2}}{R_{1}}
$$

where constant coefficients $\mathrm{A}_{1}, \mathrm{~A}_{2}$ and $\mathrm{A}_{3}$ are expressed through the moving liquid parameters and equal to

$$
\begin{aligned}
& A_{1}=\frac{2 \cdot \Delta P \cdot \pi}{L \eta}\left\{\frac{1}{3} C_{1}(1-\sigma)-\frac{1}{\sigma}\right\} \\
& A_{2}=\frac{\Delta P \cdot \pi}{L \eta}\left\{-C_{1} R_{1}(1-\sigma)+\frac{R_{1}}{\sigma}+\frac{1}{2 \sigma^{2}}\right\} \\
& A_{3}=\frac{\Delta P \cdot \pi \cdot R_{2}^{2}}{L \eta \sigma^{2}}
\end{aligned}
$$

Relationship ( 14 ) is obtained at the next approach

$$
\ln r \approx r-1
$$

higher - order terms in this expansion are neglected. Comparison of the formula (14) with appropriate expression for incompressible liquid allows to conclude, during motion of compressible liquid in cylindrical pipe flowrate will be sufficiently less under otherwise equal conditions. 


\section{HYDRAULIC LOSSES AT MOTION OF COMPRESSIBLE LIQUID}

Compressibility of liquid by law ( 2 ) leads to changing the coefficient of hydraulic resistances $\kappa$ along coordinate $z$. How it is known

$$
k=64 / R e
$$

and taking into consideration $R e=\rho v d / \eta$, for coefficient $\kappa_{\text {comp }}$ of compressible liquid accordingly to distribution ( 2 ) we have

$$
k_{\text {comp }}=k_{0} e^{\lambda z}
$$

where $\kappa_{0}$ - coefficient of resistances for the same liquid, if it would be incompressible. Naturally, head loss $\Delta \mathrm{h}$ by $\mathrm{D}^{\prime}$ arcy determined like

$$
\Delta h=\int_{0}^{H} k_{\text {comp }} \frac{v^{2}}{2 g d} d z
$$

increases also. We obtain

$$
\Delta h=k_{0} \frac{v^{2}}{2 g d} \int_{0}^{H} e^{\lambda z} d z=\frac{k_{0} v^{2}}{2 g d \lambda}\left\{e^{\lambda H}-1\right\}
$$

in the last integrating $v$ and $d$ are assumed to be permanent as simplifying circumstances. Comparison of formula ( 16 ) with known head loss for incompressible liquid

$$
\Delta h_{0}=k_{0} \frac{v^{2}}{2 g d} H
$$

exposes, that the losses arise by the value

$$
\omega=\frac{\Delta h}{\Delta h_{0}}=\frac{e^{\lambda H}-1}{\lambda H}=1+\sum_{j=1}^{\infty} \frac{(\lambda H)^{j}}{(j+1) !}
$$

thereto, the greater compressibility degree, in other words, parameter $\lambda$, the more essential head loss on section $H$ will be. Formulae ( 15$)-(17)$ are valid for laminar section of liquid motion only, when coefficient of hydraulic losses $\kappa$ depends on the liquid rheological characteristics. On turbulent section $\kappa$ is proposed to be constant, and within the last proposition head losses $\Delta \mathrm{h}$ for compressible and incompressible liquids are equivalent.

Strictly saying, in deducing formula ( 16 ), it would be necessary to keep in mind dependence $\eta=\eta(z)$ because of solubility of solid phase in liquid. It is well - known, if small solid particles are weighted in liquid ( like rock pieces in drilling mud ), then coefficient of intrinsic friction increases due to this factor by value proportional to ratio of full volume of solid phase to liquid general volume. In [ 12 ] it was obtained the formula taking into account this correction with accuracy till the first degree of concentration terms, assuming for mud to be homogeneous ( by radius ) disperse system - condition available for us and taken in the beginning of this article, namely

$$
\eta^{*}=\eta(1+2,5 \varphi)
$$

here $\eta^{*}$ - solution ( mud ) viscosity, $\eta$ - viscosity of pure solvent ( water, if drilling mud is based on water, and/or oil for oil emulsion ), $\varphi$ - volume concentration defined as ratio of drilled out rocks volume to liquid that; the question is clearly considered in [ 10$]$. Since in 
real conditions $\varphi=\varphi(z)$, then the last aspect should effect on head losses determined by the relationship (16).

Taking into consideration, that volume concentration alters with depth by law identical to (2)

$$
\varphi(z)=\varphi_{0} e^{\lambda_{1} z}
$$

$\varphi_{0}$ - volume concentration on well-head, after calculations we get, using for simplicity $\lambda_{1} \approx$ $\lambda$

$$
\Delta h=\frac{k_{0} v^{2}}{2 g d \lambda}\left\{\left(e^{\lambda H}-1\right)-\sum_{j=2}^{\infty}(-1)^{j} \frac{\varphi_{0}^{j-1}}{j}\left(e^{j \lambda H}-1\right)\right\}
$$

Comparison with equivalent incompressible liquid gives

where $\omega$ is found by (17).

$$
\frac{\Delta h}{\Delta h_{0}}=\omega-\frac{1}{\lambda H} \sum_{j=2}^{\infty}(-1)^{j} \frac{\varphi_{0}^{j-1}}{j}\left(e^{j \lambda H}-1\right),
$$

Calculated so far losses are justified at liquid motion in pipe. If liquid compressible by ( 2 ) moves in borehole annulus, then pressure losses by $\mathrm{D}^{\prime}$ arcy will be respectively equal to

$$
P_{D}=\frac{8,44 \cdot 10^{4} \cdot g Q}{\left(R_{1}-R_{2}\right)^{4}\left(R_{2}+R_{1}\right)^{2} \cdot v} \int_{0}^{H} \eta(z) d z
$$

for laminar flow, and

$$
P_{D}=\frac{82,6 \cdot k \cdot g Q}{\left(R_{2}-R_{1}\right)^{3}\left(R_{2}+R_{1}\right)^{2}} \int_{0}^{H} \rho(z) d z
$$

for turbulent one; herein $\mathrm{Q}$ - constant flowrate through cross - section ( more detailly this question is investigated in [ 7 ]. Under known dependences $\eta(z)$ and $\rho(z)$ (see above) calculations of integrals can be realized without problems.

\section{SUPPLEMENTARY FACTORS EFFECTING ON COMPRESSIBLE LIQUID MOTION}

Law ( 2 ) and, naturally, all the computations made in previous chapters are reasonable for isothermal liquid motion only. In this matter, they are available for drilling ( in general, any liquid transportation ) within such intervals only, where temperature is estimated to be constant. If the last condition is not valid, then instead of above dependence

(2), we should use more general distribution listed in [4]

$$
\rho(z)=\rho_{0} e^{-\lambda z / T(z)}
$$

where $T(z)$ is known function

$$
T(z)=T_{0}+\gamma z
$$

hereafter $T_{0}$ - mud temperature on well-head, $\gamma$-thermal coefficient for ascending liquid flow. So, for mud density we ultimately have

$$
\rho(z)=\rho^{*} e^{\alpha / \beta+z}
$$


where next abbreviations are used

$$
\rho^{*}=\rho_{0} e^{-\lambda / \gamma}, \alpha=\lambda T_{0} / \gamma^{2}, \beta=T_{0} / \gamma
$$

Taking into account distribution ( 22 ), from the continuity equation the following relationship is concluded

$$
\operatorname{div} v=\frac{\alpha}{(\beta+z)^{2}}(v-w)
$$

From ( 23 ) it should be noted, for term grad div $v$ we get magnitude depending on $z$. In other words, liquid velocity is function of coordinate $z$ as well, i.e. $\partial v / \partial z \neq 0$ already, hence we are not able to neglect non- linear summand connected to $v(\partial v / \partial z)$ in Navier Stokes equation. So, for account of temperature during compressible liquid motion we should resolve non - linear equation. Nevertheless, in some cases, when $z<<\beta$ (condition of quasi - isothermal approximation ), div $v$ does not depend on $z$, how it can be seen from ( 23 ), and calculations made in chapters $\mathbf{2 - 5}$ are formally usable for mud ( liquid) moving in pipe with temperature gradient. Having used empirical values of $\mathrm{T}_{0}$ and $\gamma: \mathrm{T}_{0}=290 \mathrm{~K}, \gamma=0,015 \% \mathrm{~m}$, for parameter $\beta$ we have next estimation $\beta \approx 2 \cdot 10^{4} \mathrm{~m}$. That's why, for middle wells of depth $h \leq 2000-2500 \mathrm{~m}$ present calculations may be used, because $\mathrm{z} / \beta \approx 0,1$. For deeper wells conclusions of the article are usable with certain inaccuracy, that to be found in every separate problem. In realizing drilling process during warmer season, this limit will increase due to higher temperature of surrounding medium on well-head $\mathrm{T}_{0} \approx 300-305 \mathrm{~K}$, so the calculations may be used with the same accuracy for wells with depth $\mathrm{h} \leq 3000 \mathrm{~m}$. In other words, the higher temperature of surrounding medium, the greater height drops for which results obtained here might be used.

Another factor. which able to change the result is so - called source function characterizing sludge generation degree on bottomhole during bit action. It is generally taken, in the equation of mass transfer in borehole annulus while drilling (if diffusion flow is negligible in comparison with connective one )

$$
\frac{\partial(\Delta \rho)}{\partial t}+v \frac{\partial(\Delta \rho)}{\partial z}=f(z, t)
$$

the right side is proposed to be zero. However, such an approach is not correct, because by physical matter drilling process means continuous increment of sludge mass due to bit functioning, so while drilling process $f(z, t)$ can not be equal to zero in principle, but should change by certain law determined with the process parameters. Undoubtedly, in connection with this aspect the continuity equation obtained from mass balance should be changed also. Now it is not clear, how it will be for drilling, but one circumstance is obvious : this equation should consist of all the drilling parameters.

\section{CONCLUSION}

In current paper it has been investigated the hydrodynamic changes, which can appear during motion of liquid compressible by exponential law ( 2 ) between two cylindrical pipes. Described variant is available for drilling mud motion in borehole annulus. However, it is absolutely obvious, that developed method may be used for motion of liquid with any compressibility law along cylindrical pipe also, if integration limits are changed to be 
$0 \leq \mathrm{r} \leq \mathrm{R}$. In the indicated approach our calculations are usable for problem of liquid transportation across dissected area with height difference no more, than $\delta \mathrm{h} \approx 2500 \mathrm{~m}$. Practical calculations made for real transportation pipeline [ 13 ] by $R$. Groombridge initiative, Pipeline Manager - Kvaerner John Brown, show, that in this case compressibility of transported agent (first of all, oil and gas ) is estimated to be sufficient and should be naturally taken into consideration. Question about compressibility of transported liquid and/or gas is firstly concerned in present paper, so in the light of hydrodynamic computations made here its resolving is greatly urgent.

\section{REFERENCES}

1) L.I. Turchak and V.R. Shydlovski, About motion equation of stratified liquid, Doklady Akad. Nauk SSSR 2, 254, 317 - 321, 1980.

2) V. M. Kamenkovich, Principles of ocean dynamics, Gidrometeoizdat, Leningrad ( in Russian ), 1973.

3) O. F. Vasilyev et al, Annals of sciences, Hydromechanics, Moskva 8, 120 - 128, 1975.

4) H. G. Hassanov, Geophysical parameters impact upon borehole drilling mud density and hydrostatic pressure, Geophysics news in Azerbaijan 2, 20 - 22, 2000.

5) H. G. Hassanov, Workshop Offshore Caspian Drilling, October 2000, Baku

6) L. D. Landau, E. M. Lifshitz, Hydrodynamics, Nauka, Moskva, 1986.

7) H. G. Hassanov, Ways for improving the drilling process by barometric index, Preparing for publication, Khazar University Press, Baku, 2001.

8) E. Kamke, Handbook on differential equations (in Russian ), Nauka, Moskva, 1976.

9) L. J. Slater, Confluent hypergeometric functions, In : Handbook on mathematical functions, Ed. by M. Abramowitz and I. Stegun, National Bureau of Standards, Applied Mathematics Series - 55, New - York, 1979.

10) N. Makovey, Hydraulics of drilling, Nedra, Moskva, 1986.

11) A. Kh. Mirzajanzadeh et al, Hydraulics of drilling and cement muds, Nedra, Moskva, 1966.

12) A. G. Grossman, Statistical questions of mechanics and heat conductivity of suspension, Thesis, Ph.D., Novosibirsk, 1978.

13) H. G. Hassanov, Possibility of practical using of electric field influence on liquid viscosity in transportation systems, accepted for publication in $M C A$ 
